traçant la circonférence $\mathbf{Q R S}$ tangente à la bissectrice des vecteurs $(x, \varphi)$, à :

$$
\overline{2 \mathrm{OU}^{2}} \leq \overline{\mathrm{OS}^{2}} \quad \text { ou } \leq \frac{\mathrm{OS}}{2}
$$

La vérification préalable est donc des plus simples. Il suffit de tracer la bissectrice des vecteurs $(\alpha, \vartheta)$ et de déterminer 'le' point de tangence sur cette droite, de la circonférence passant par $\mathbf{Q}, \mathbf{R}$. Si on projette d'autre part sur cette bissectrice la droite $\mathbf{O V}$ rabattue au préalable sur le vecteur ç, la projection $\mathbf{U}$ de l'extrémité doit se trouver en deça du milieu de $\mathbf{O S}$.

De ce qui précède, il résulte aussi que s'il existe une solution correspondant à un point $\mathbf{C}$, il en existe une seconde correspondant au point $\mathbf{C}$ ', symétrique de $\mathbf{C}$ par rapport à la bissectrice $(\alpha, \varphi)(\mathrm{r})$.

Les deux solutions se confondent pour $\mathrm{OU}=\frac{\mathbf{O S}}{2}$, c'esta-dire quand :

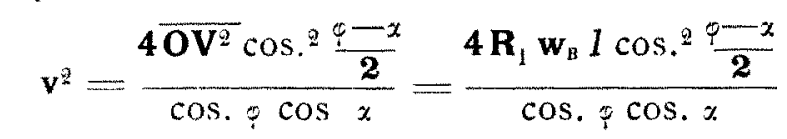

$\mathbf{v}=:=2 \cos . \frac{\varphi-x}{2} / \frac{\overline{\mathbf{R}_{1} \mathbf{w}_{B} I}}{\cos , \varphi \cos . x}=\mathbf{2} \cos \cdot \frac{\frac{\rho-\alpha}{2}}{\frac{\mathbf{w}_{\mathrm{B}} \mathbf{R}}{\cos . \varphi \cos . x}}$

La valeur correspondante de $\mathbf{u}$ se déduit immédiatement de la figure ci-dessous :

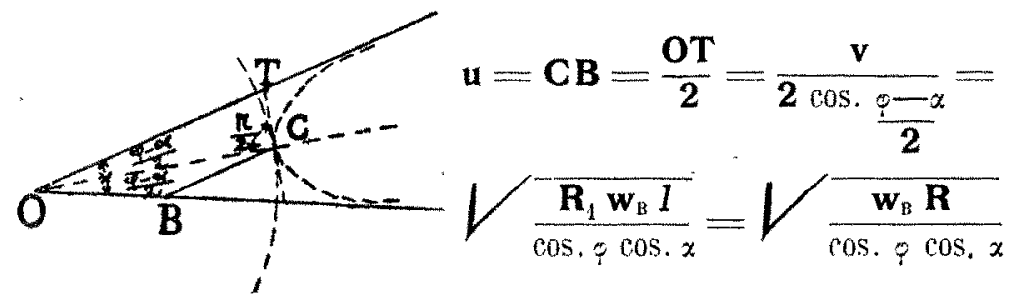

Il est facile de la déterminer géométriquement en se rappelant que $\overline{\mathbf{O V}^{\mathfrak{2}}}=\mathbf{R}_{1} \mathbf{w}_{\mathrm{B}} l=\mathbf{w}_{\mathrm{B}} \mathbf{R}$

D'où, fig. 7 :

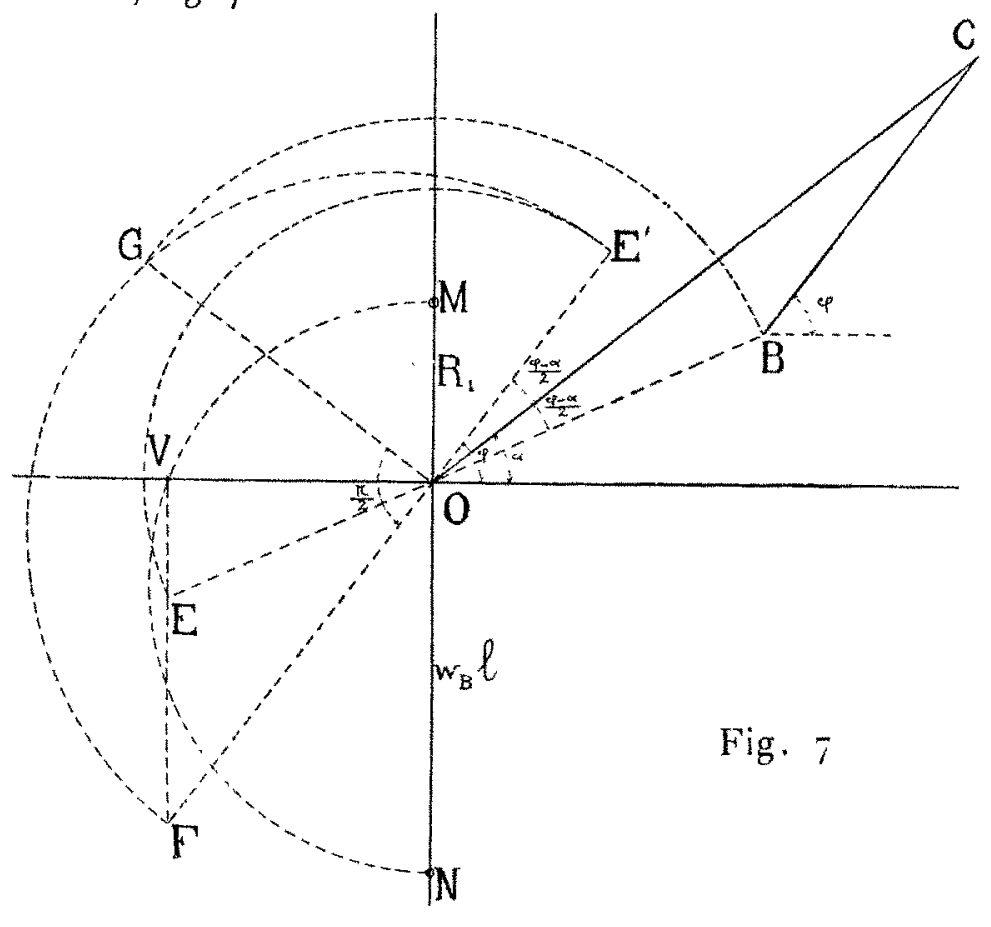

(I) De ces deux solutions une seule est généralement pratique, la seconde conduisant a des densités de courant inadmissibles.

$$
\begin{aligned}
& \mathbf{u}^{2}=\frac{\overline{\mathbf{O V}^{2}}}{\cos . x \cos . \tau}=\frac{\overline{\mathbf{O V}}}{\cos . x} \times \frac{\mathbf{O V}}{\cos . \phi}=\mathbf{O E} \times \mathbf{O F}=\mathbf{O E} \times \mathbf{O F}=\overline{\mathbf{O G}^{2}} \\
& \mathbf{u}=\mathbf{o G} \text {. }
\end{aligned}
$$

En rabattant $\mathbf{O G}$ sur $\mathbf{O B}$ et traçant de $\mathbf{B}$ la parallèle de direction $q$ jusqu'à sa rencontre avec la bissectrice des vecteurs $(\alpha, \varphi)$, on détermine la valeur minima $\mathbf{O C}$ de $\mathbf{v}$ qui rend le problème possible, et pour laquelle les deux valeurs de $\mathbf{u}$ sont confondues en une seule, égale à $\mathbf{O G}$ ou $\mathbf{B C}$ (I).

C'est un second moyen de vérification, puisque la valeur donnée de $\mathbf{v}$ doit être supérieure à la valeur ainsi déterminée de $\mathbf{O C}$.

$$
\text { P. Dumas, }
$$

Ingénieur des Ponts et Chaussées.

\section{Un moyen de dresser le Catalogue des chates d'eau de France}

"Ingénieurs, industriels.... travaillons surtout nous" mêmes à l'œuvre dont nous voulons bénéficier ", tels sont les termes du ralliement que $M$. Côte a cru devoir sonner dans le no 1 de la Houille Blanche, à toutes les bonnes volontés que peut grouper l'étude des forces hydrauliques de notre pays.

Nous avons entendu cet appel et nous venons faire une proposition quant aux voies et moyens par lesquels se pourrait réaliser le catalogue si désiré de nos forces hydrauliques.

Dans les premières pages de son très remarquable ouvrage, M. R. Tavernier a tout naturellement proposé de charger de ce soin un nouveau cadre administratif, et il ne faut pas nous en étonner. N'est-ce pas une manière bien française de comprendre les choses? Pourtant, quand on réfléchit aux résultats de la centralisation administrative dans les ceuvres de notre pays, on se prend au désir d'essayer d'une autre solution.

Assurément, les travaux que ce nouveau rouage administratif produiraient seraient marqués au cachet de la probité scientifique la plus rigide et, par avance, sans aucune flatterie, on peut dire qu'une fois achevés ils constitueraient un monument impérissable.

Mais à quelle date verrait-on cet achèvement ? La besogne est complexe et, pour la mener promptement à terme, il faut un personnel considérable. Considérable aussi serait la dépense, et, sans faire aucune incursion dans la politique, il est à prévoir que l'Etat, malgré son bon vouloir indiscutable, ne trouvera pas les ressources nécessaires pour aboutir vite. Or, ce n'est pas dans un siècle que l'enquête doit aboutir, mais demain, après-demain au plus tard.

Et puis les résultats de ces travaux veulent être publiés pour avoir leur valeur vraie. Sans doute, l'Etat fait des

(1) Comme dans ce cas $\mathbf{B C}=\mathbf{O B}=\boldsymbol{Z I}(\boldsymbol{Z}$ impédance d'un fil de ligne), il en résulte que la tension à larrivee est ègale à la chute de tension dans un filde ligne. 
publications et est outillé pour donner des renseignements à qui vient les lui demander. Cependant le public, qui a évidemment le plus grand tort de méconnaître les ressources que l'Etat met à sa disnosition, s'abonne peu au Joumal Officiel et ne sait pas faire ces demandes. Il regarde toujours à deux fois avant d'aller affronter une administration à fins de renseignements: la solennité officielle l'effarouche. S'il $s^{\prime} y$ hasarde il se heurte à des fonctionnaires, très courtois évidemment, mais dont le temps précieux appartient à l'Etat et ne peut pas s'accommoder de questions imprécises. Le questionneur est alors dans le plus grand embarras pour préciser à autrui ce qui, par le défaut même de renseignements préalables assez nombreux, n'est pas encore très net dans son esprit. Il se retire découragé et le travail des uns comme l'initiative de l'autre aboutissent à un résultat stérile, temps et argent ont été dépensés en pure perte.

Ce sont là des inconvénients pour ainsi dire constitution. nels qu'entraine avec elle toute organisation trop centralisée. Du reste, notre pays n'en a pas le monopole, on les observe aussi à l'étranger; partout aux mêmes causes répondent les mêmes effets.

Il nous a semblé qu'une association des initiatives privées, mises en jeu par l'étude du problème de la Houille blanche, pouvait obvier à la majeure partie des défauts que nous venons de signaler. L'époque semble propice pour 1enter un pas dans cette voie. Plusieurs syndicats se sont déjà constitués soit pour renseigner leurs adhérents sur les Huctuations des marchés, soit pour soutenir leurs revendications devant les pouvoirs publics, soit aussi, quelques-uns poussant plus loin que la réalisation immédiate des aflaires, poùr ćtudier les progrès, dont sont susceptibles la créatiôn, l'aménagement et l'exploitation des forces motrices hydrauliques.

Quand des intérêts sont ainsi conscients d'eux-mêmes, ils sont bien près de triompher des obstacles opposés à leur cxpansion. Or, tous les membres de ces associations ont, des maintenant, peu ou prou des renseignements sur les forces hydrauliques qu'ils possèdent, qu'ils exploitent, même sur celles qu'ils voient dans leur voisinage. Si on leur donnait le moyen de faire connaître, sans aucune fatigue appréciable pour eux, sans aucun débours, les renseignements qu'ils ont en leur possession, et qu'on leur fît observer qu'en échange de leur livraison à la masse, ils bénificieront de l'apport de cette masse, nous pensons qu'un grand point serait déjà acquis.

Un second effort consisterait dans la coordination de tous les renseignements suivant un classement méthodique, puis dans leur publication.

Or, ce sont là des cuvres qu'un périodique comme la Houllle Blanche est plus qualifié que personne pour entreprendre... C'est partie intégrante de son programme même...

Il est tout clair qu'on ne saurait obliger personne à livrer ses renseignements, mais nous pensons que lorsque les intéressés auront bien pénétré les avantages de la mutualité en matière de renseignements techniques, les récalcitrants seront rares.

On ne manquera pas d'objecter qu'on n'aura ainsi que des renseignements sur des installations déjà faites ou entre les mains de possesseurs qui les utilisent industriellement, tandis que l'intérêt majeur va aux installations à innover.

Il en pourra être ainsi en effet au premier moment, mais rien ne dit que la doive se borner l'effort! L'essentiel est de commencer, le développement ne semble pas devoir être douteux.

Et puis l'argument nous semble un peu spécieux, la propriété change de mains et se modifie, et ce sont là des événements qui importent. N'est-ce donc rien, quand on veut acheter une usine existante, que de savoir qui on a au-dessus ou au-dessous de soi et quelles sont les possibilités du cours d'eau sur lequel on médite de s'installer ou d'acquérir une usine. N'est-il pas évident que le marché se présentera avec des avantages ou des inconvénients variés, selon qu'on saura pertinemment d'avance, et par dires contradictoires, qu'on peut ou non augmenter son installation dans une limite ou une autre, qu'on sera affranchi de servitude ou, qu'au contraire, on se verra grevé ? - Sans doute ce sont des points dont on s'enquiert quand on veut conclure une affaire, mais cette enquête comporte frais, démarches, usure de temps, n'est-ce donc rien que d'aroir ces renseignements dès le premier instant et avec un minimum d'effort? La réponse n'est pas douteuse et voilà les premiers fruits que peut porter l'enquête privée que nous convions la Houille Blanche à commencer sans retard. Après tout, cela vaut mieux que de rester à se lamenter sans rien faire.

Les renseignements fournis par les collaborateurs volontaires à cette enquête peuvent, à la rigueur, être donnés sous une forme quelconque, pourvuqu'elle soit claire et précise. Il nous a semblé que le travail de leur mise en œure serait bien simplifié si chacun voulait bien s'astreindre à la discipline d'observance facile qui consiste à tracer et à remplir des tableaux conformes aux modeles ci-joints appuyés de feuilletons de la carre d'Etat-major (1) $\left(\frac{1}{80.000}\right.$ ou $\left.\frac{1}{50.000}\right)$ ou de tout autre service pourru qu'elle fut claire.

Nous inscrivons sur la feuille de modèle les recommandations principales qui doivent être présentes à l'esprit des rédacteurs pour faciliter le travail de dépouillement et de classement.

Tous les trois mois, ou plus souvent si cela devenait nécessaire, la Houille Blanche publierait le résultat des renseignements reçus dans le trimestre sous la forme de tableaux identiques et, enfin, annuellement une mise à jour en pourrait être faite en vue de la réunion en un seul volume des résultats produits au courant de l'année.

Bien que nous ayons plus spécialement en vue les eaux qui sont du domaine privé des citoyens, done dans la partie de leur cours située en amont des points où les cours d'eau sont flottables et navigables, nous croyons que cette statistique pourra utilement comprendre aussi des renseignements sur les parties aval. Sans doute, l'Administration des Ponts et Chaussées a la haute main dans cette région et y poursuit un travail analogue à celui que nous préconisons ici; mais, outre que le grand public n'est pas initié au résultat de ses travaux, il peut être utile, pour tout le monde de provoquer

(1) o fr. 3o à I fr. selon l'éche'le, chez tous les libraires. 
MODELE DE TABLEAU POUR UN COURS D'EAU

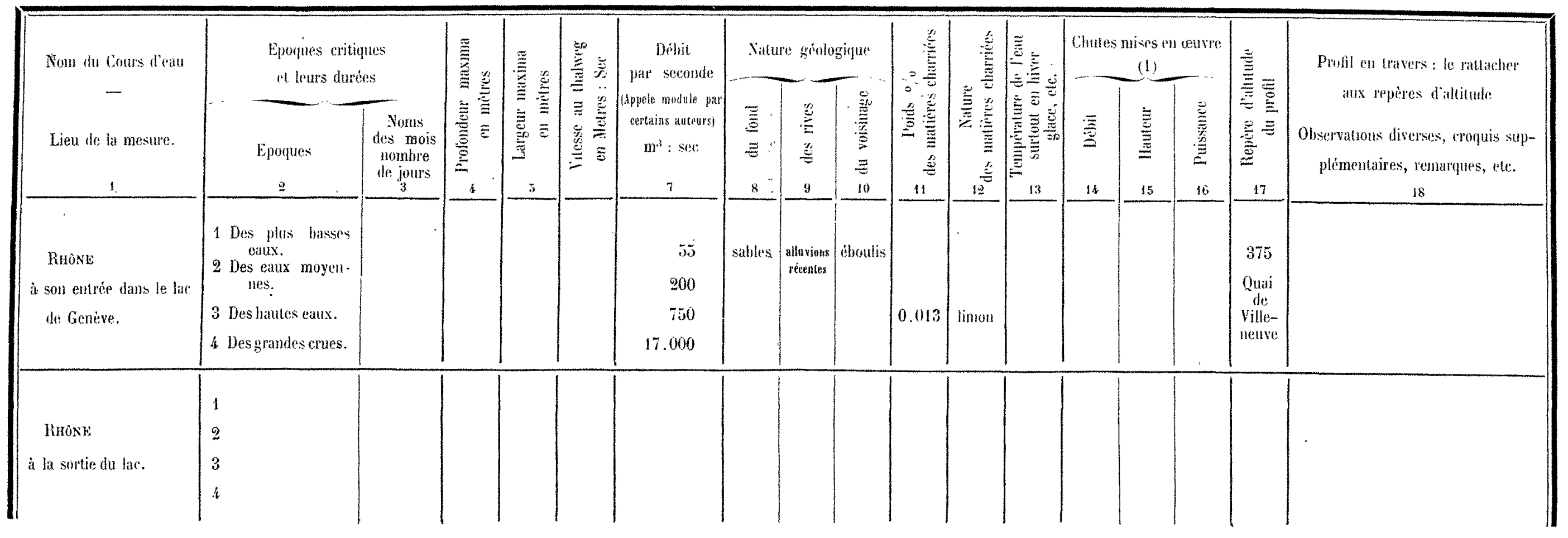

MODELE DE tABLEAU POUR UN LAC

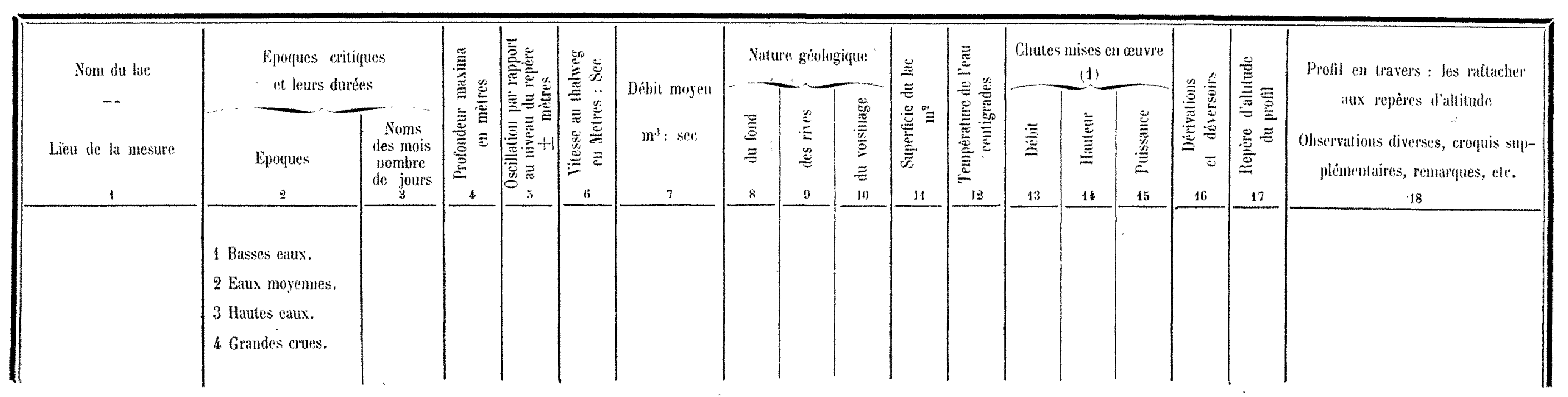

(I) Quand il y aura lieu de signaler des chutes, bien spécitier et marquer sur la carte quéls sont les points de départ el l'aboutissement de la colonne d'eau.

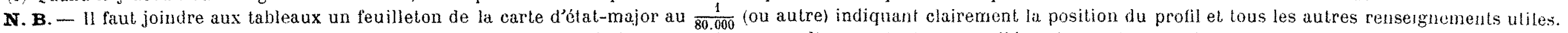

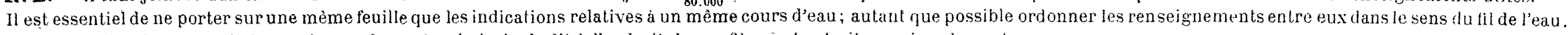
A défaul de la vilesse au thalweg, donner la pente générale du lit à l'endroit du profil; pente et vilesse si on le peul. 
l'éclosion de renseignements obtenus par des observateurs s'ignorant mutuellement: la vérité ne peut qu'y gagner. L'un de nos modèles de tableaux est relatif aux cours d'eau, l'autre aux lacs et étangs qui, même les plus petits, peuvent être fort intéressants. 11 y aurait grand avantage à faire rentrer dans cette catégorie les lacs artificiels provenant de retenues d'eaux, soit déjà faites, soit projetées, soit simplement possibles... Les meilleurs cours d'eau, au point de vue mécanique, sont ceux qui, s'alimentant à un glacier ou à un lac, à ciel ouvert ou souterrain, ne tarissent jamais. Cette enviable disposition est rare et il est bien évident que dans l'avenir on fera tout ce qu'on pourra pour la créer artificiellement là où la nature ne l'a pas mise expressément.

Enfin, il n'est pas nécessaire d'attendre qu'on ait réponse à toutes les questions du tableau pour remplir celui-ci et l'envoyer à la Houille Blanche. Qu'on envoie d'abord ce qu'on a, le reste suivra à son heure.

Telle est la manière dont, à ce qu'il nous semble, l'industrie française peut, à peu de frais et dans le moindre temps, réunir des renseignements dont elle a le plus impérieux besoin. Nous serions heureux, si nous pouvions faire partager nos vues par nos lecteurs, et s'ils consentaient à devenir nos collaborateurs dans cette voie.

\section{Commandant Andebravd, Ancien élève de l'Ecole Polytechnique ingeniaur}

POST-SGRIPTUM. - On nous a fait observer que le travail que nous proposons d'édifier a la plus grande analogie avec celui que M. Lauterburg a dressé pour la Suisse, qui a été reconnu insuffisant et que le Service des.Travaux Publics helvétiques a remplacé par une enquête oú interviennent "l'étendue et la topographie du bassin de * réception. Des jaugeages, en effet, pour donner des indications cer" taines, doivent être multipliés à l'infini et prolongés pendant une " très longue suite d'années. La connaissance exacte du bassin de - réception peut, au contraire, donner immédiatement des aperçus - très précieux sur la capacité et la régularité de l'alimentation du "cours d'eau... (R. Tavernier. Les forces hydrawliques des Alpes, page 24.)

Ce sont là idées auxquelles nous sommes tout acquis Nous espérons le prouver dans une prochaine communication à la Houille Bilanche; mais qu'on veuille bien remarquer que : $1^{\circ} \mathrm{La}$ connaissance topographique que nous avons de notre Massif alpin est loin d'avoir la même précision que celle que les Suisses ont du leur. Pour des raisons de temps et d'argent, notre carte d'Etat-major, dont la confection fut poursuivie depuis 1827 (abstraction taite de la période de I 817 à 1827 ), sur un sol douze fois plus grand que celui de la Suisse, par un personnel relativement peu nombreux, ne comporte guère qu'une cote d'alticude géométriquement établie par kilomitre carré, quand la carte suisse du général Dulour (ancien élève de l'Ecole polytechnique française) en comporte 60 ou 80 . Cette dernière a pu être levée entièrement à la planchette, la carte de France a dû, pour ne pas exiger trois ou quatre siecles pour sa confection, se borner à raccorder les cadastres et à inscrire sur ses mappes des figurés de terrain, faits a vue le plus souvent.

Si nous citons ces faits, ce n'est pas pour dénigrer cette cuvre, bien au contraire. On la tient pour unchef-d'auvrequand on compare le résultat à la pauyreté des morens mis enjeu el il ne faut pas mènager l'admiration à ses auteurs, ce serait une révoltante injustice. Mais, il était dans la force des choses que ce résultat fut imparfait et, notamment pour le problème d'hydraulique que nous visons ici, on ne peut accepter les indications de cette carte que sous bénéfice d'inventaire. Or, au point de vue du figuré du terrain, c'est la seule carte sérieuse que le public ait à sa disposition. L'application à notre cas de la méthode des Travaux Publics helvétiques exigerait donc qu'on refit d'abord une carte de nos Alpes. Les Suisses ont mis 32 ans pour faire la leur! Nous sommes trop pressés pour attendre un si longtemps.

Au surplus cette connaissance topographique n'aura toute sa valeur que lorsque la connaissance géologique (en bonne voie d'avancement) de la surface sera achevée et surtout lorsque l'interprétation des faits topographiques et géologiques pourra s'é.lairer de la connaissance des lois principales de la Météorologie. Or, cette dernière science, vieille d'à peine cinquante ans, n'a pas encore des fondements indiscutables.

$2^{0}$ Notre enquête n'empêche nullement de refaire l'enquête topographique, indispensable nous le répétons, mais dont le résultat ne peut ètre qu'à longue échéance.

Par notre moyen nous aurons une première approximation. Or les solutions industrielles ne s'acquièrent que par des approximations successives. Ingénieurs et industriels habitués à cette méthode de recherches ne peuvent donc sérieusement répudier son aide.

3o N'ayant rien pour le moment nous n'avons pas le droit d'ètre difficiles, sous prétexte que l'offre première est trop maigre. Un tiens valant mieux que daux tu l'auras, prenons d'abord ce qui est offert puisque cela ne nuit nullement à la réalisation d'un avenir meilleur qu'on attendra plus patiemment.

$4^{\circ}$ Enfin, l'cuvre topographique n'est possible qu'avec un personnel et des fonds considérables. Un jour viendra peut-être et, pour notre compte, nous fuisons des vœux pour le voir luire bientôt, ou l'inıtiative de l'industrie privée de notre pays se haussera à la réalisation d'œuvres telles que le lever et la publication d'une carte exacte des Alpes françaises. Nous sommes convaincu que nos industriels en tireraient un profit sérieux, sans parler de l'honneur, mais force nous est hien de reconnaitre qu'il coulera encore beancoup d'eau dans les torrents avant que le rêve puisse recevoir un commencement l'exécution!

Pour le moment l'Etat seul est, chez nous, en passe de faire un tel travail. Nous avons dit plus haut ce qu'il faut penser de la rapidité avec laquelle il peut aboutir. $\mathrm{C}$. A.

\section{NOTE DE LA RÉDACTION}

La Houille Blanche acceptant, pour réaliser son programme, tous les concours compétents et désintéressés, remercie M. le commandant Audebrand de sa collaboration. Si, dans le but de perfectionner la méthode proposée, des critiques fondées lui sont faites, d'où quelles viennent, il en sera tenu compte. Quant à nous, croyant que le mieux est de juger la méthode à l'emploi, nous sommes prèts à l'appli. quer et nous invitons nos lecteurs à nous demander des tableaux imprimés d'après les modèles ci-dessus; ils seront envorés gratuitemeut.

La Revue coordonnera,suivant un c'assement méthodique, tous les renseignements qui lui parpiendront parce moren et elli. les publiera périodiquement.

\section{LES ÉTUDES ÉLECTROTEGHNIQUES}

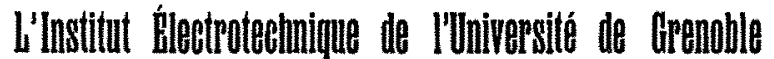

\section{(SUITE ET FIN)}

L'œuvre d'enseignement de l'Institut ne s'exerce pas seulement au profit de sa clientèle spéciale d'étudiants. Elle s'étend aussi au public grenoblois par un cours du soir que patronnent et rétribuent la Ville de Grenoble et le Conseil général de l'Isère ; elle intéresse enfin un cercle de correspondants toujours plus étendu en France et à l'étranger, 